\title{
Examining the Influence of Different Physical Activity Training on the Postural Stability of University Students
}

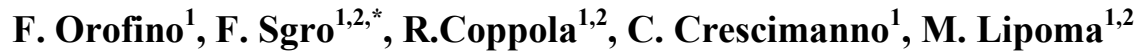 \\ ${ }^{1}$ Faculty of Human and Social Sciences, University of Enna "Kore", Italy \\ ${ }^{2}$ Laboratory of Movement and Performance Analysis, University of Enna "Kore", Italy
}

Copyright (C) 2015 by authors, all rights reserved. Authors agree that this article remains permanently open access under the terms of the Creative Commons Attribution License 4.0 International License

\begin{abstract}
Static balance ability has a critical role in the execution of complex motor patterns as well as normal daily activities, and its efficiency could be improved by means of a regular and specific training program. In this study we investigated the relation between postural stability and different types of physical activities (PA) training among university students. Thirty-six students were enrolled and grouped into two PA categories: the Exergames category included subjects who use Nintendo Wii Balance Board gaming at least twice a week, and the Sport category included subjects who practiced sport activities at least twice a week. Subjects who did not practice structured sports or physical activities were enrolled as Control. Postural control was assessed by means of a normal standing balance test with open eyes (OE) and closed eyes (CE), respectively; a force plate was used to acquire data of the center of pressure (CoP) sway. From the CoP sway we were able to select two variables: the total displacement of sway (DOT) and the mean velocity. The mean velocity was estimated for antero-posterior $\left(\mathrm{MV}_{\mathrm{AP}}\right)$ and medio-lateral $\left(\mathrm{MV}_{\mathrm{ML}}\right)$ directions, respectively. ANOVA revealed significant differences among groups for the three variables. We found that the mean of DOT and $\mathrm{MV}_{\mathrm{AP}}$ was significantly decreased in the Exergame's category compared with the others groups. Furthermore, the differences were higher for the $\mathrm{CE}$ than the $\mathrm{OE}$ test. The training programs based on the use of exergames seems to improve the postural control level of the aforementioned ability more than conventional sport training.
\end{abstract}

Keywords Postural Stability Assessment, Balance Training Programs, Sport Training, Exergames Training

\section{Introduction}

Balance is closely linked to the normal activities that everyone carries out every day: standing, sit-to-stand, and sport performance. Balance is one of the main components of coordinative abilities [1] and it can be defined as the ability to maintain the body's center of gravity within its base of support [2]. This ability can be categorized as either static or dynamic balance [2]. Static balance is the ability to maintain a base of support with minimal movement, while the dynamic balance is the ability to perform a task while maintaining a stable position [1]. Although both typologies of ability are valuable, the static balance is the main type of balance assessed both in youth and in elderly people.

Several factors and systems influence this ability, such as the visual, somatosensory, and vestibular systems. These receive sensory information streams from distal receptors into the central processing regions of the central nervous system that process and activate motor responses. Muscles, tendons, ligaments, and joints concur to maintain both static and dynamic balance. Upper and lower limbs are involved in maintaining an upright position and to correct the posture during dynamic tasks (i.e., gait cycle), but the lower ones have a critical role in maintaining standing static balance because they support body's weight and allow bipodal posture. In their actions, the strategies used are the ankle strategy, which involves the muscles for flex and extension of this joint (i.e., soleus, gastrocnemius, tibialis anterior), and the hip strategy, which acts mainly on the sway of the center of mass (CoM).

The evaluation of balance cannot be separated from the action of which it is an integral component, or from the environment in which it is performed [3]. Likewise, the characteristics of a task and/or the environment constraints may influence balance performance. The vision system is the most important sensory channel to control the postural stability of healthy subjects [22] and it plays an obligatory role in the processing and integration of other sensory inputs [23]. The role of vision in postural stability was addressed in athletes of rugby, soccer, and sprinter [5]. The authors argued that the absence of vision has more impact on the balance performance of the rugby athletes that of the soccer practitioners. The critical role of the vision in the postural stability of several sports athletes was also supported by a recent systematic review [24]. 
The postural stability could be improved with different exercises and training programs. Recently, the investigation on the balance abilities was mainly based on the comparison of different typologies of training programs related to traditional exercises, to the exercises included in traditional sport-based training program, and to an innovative training program based on the use of the exergames. Ricotti assessed balance abilities among young soccer players involved in different typologies of training programs. These programs were built unifying soccer exercises with break-dance and swimming exercises, separately [4]. Hammami and colleagues showed that static balance performances were sports-dependent, while general sport activities were not important for improving that ability [5]. In addition, those researches suggested that the repetitive training exercises improved balance abilities.

The exergames are videogames where the players need to move their body to interact with the game. The aim of these tools is to combine fun, fitness, and well-being in order to promote the physical activity during game play and to fight the growth of obesity. Among these tools, the Nintendo Wii Fit exercise-based games were widely used for the training and the assessment of the balance ability in children [6][7], adults [8][9][10], and the elderly [11]. The Wii Fit games use the Nintendo Wii Balance Board to gather information on the player's center of pressure sway. The Wii Balance Board (WBB) is a low-cost force plate and it has been indicated by previous studies as an amazing innovation related to the exergames [11].

Considering the findings of the aforementioned studies $[4-5,8-10]$, the balance-based exergames seem to be worthwhile for improving the balance ability rather than general sport or physical activity. Thus, we investigated the postural stability among groups of university students by means of standing balance tests with different vision conditions. In this respect, we hypothesized that the students who played balance-based exergames almost two times per week would have better postural stability performances than the participants involved in the other groups.

\section{Materials and Methods}

\subsection{Participants and Procedures}

The participants involved in this study were sampled from the students enrolled at the Physical Activities and Sport Sciences course of the University of Enna. Overall, there were 36 participants who were grouped into two physical activity categories: Exergames and Sport. The first category included the students who used WBB-based exergames at least twice a week. The second category included the students who practiced whichever typologies of sports at least twice a week. A third category (Control) was arranged with the subjects who did not practice any structured sport and/or physical activities. The characteristics of the three groups are shown in table 1 . The height was estimated using wall-mounted stadiometers, while the weight was measured by means of portable scales. The Body Mass Index (BMI) was estimated with the following formula: body mass $(\mathrm{kg}) /$ height $^{2}\left(\mathrm{~m}^{2}\right)$. All the subjects provided informed consent before the start of the study.

Table 1. Characteristics of the participants involved in the study.

\begin{tabular}{ccccccc}
\hline & \multicolumn{2}{c}{$\begin{array}{c}\text { Exergames } \\
(\mathrm{n}=12)\end{array}$} & \multicolumn{2}{c}{$\begin{array}{c}\text { Sport } \\
(\mathrm{n}=12)\end{array}$} & \multicolumn{2}{c}{$\begin{array}{c}\text { Control } \\
(\mathrm{n}=12)\end{array}$} \\
\cline { 2 - 7 } & $\mathrm{M}$ & $\mathrm{SD}$ & $\mathrm{M}$ & $\mathrm{SD}$ & $\mathrm{M}$ & $\mathrm{SD}$ \\
\cline { 2 - 7 } & 27.5 & 7.3 & 21.6 & 2.1 & 23.5 & 2.7 \\
Age (yr.) & 168.5 & 8.8 & 176.7 & 7.1 & 162.5 & 9.8 \\
Height (cm.) & 71.7 & 17.4 & 72.6 & 8.5 & 61.6 & 10.2 \\
Weight $(\mathrm{Kg})$. & 25.3 & 6.3 & 23.2 & 1.8 & 23.29 & 3.05 \\
BMI $\left(\mathrm{kg} / \mathrm{m}^{\wedge} 2\right)$ & 25.3 \\
\hline
\end{tabular}

The assessment sessions were realized in the movement analysis laboratory of the University of Enna. The participants were divided into six groups and each group entered a laboratory where two trained assessors were waiting for them. One assessor asked the participants some information about their sports experiences and acquired the data shown in table 1. The other assessor showed each group's participants the execution of the tasks required for the balance assessment. No specific wear was required for the assessment procedure, but the participants were invited to remove their shoes during the test. The assessment order was randomized assigning a random number to each participant. The assessment was realized by means of a force plate, as described in the next subsection. The Ethical Board of the University of Enna approved the methodologies and the instruments used in this study.

\subsection{Balance Assessment}

The tasks proposed for the postural stability assessment were: standing balance with the eyes open $(\mathrm{OE})$ and standing balance with the eyes close (CE). During each task the participants were instructed to keep their hands along their hips and to remain as still as they can for the acquisition time. Data were collected for 30 seconds for each task, with one minute of rest between the tasks.

The assessments were performed by means of a laboratory-grade force plate (AMTI Model OR6-7, Watertown, MA, U.S.A.). The plate's area was $46.4 \mathrm{~cm} \mathrm{x}$ $50.8 \mathrm{~cm}$ and it was calibrated following the indication provided by the manufacture guidelines. The force plate frame rate was set to $1000 \mathrm{~Hz}$, while the data of the center of pressure (CoP) sway was acquired by means of the software Vicon Nexus ver. 2.0 (Vicon, Oxford Metrics, Oxford, UK). The methodological procedures for data acquisition and for data filtering were chosen according to previous studies [12,19-21,27]. The CoP data acquired from the force plate was filtered using a low-pass Butterworth 
filter, with cut-off frequency set to $10 \mathrm{~Hz}$; from these data we were able to select two variables: the total displacement of sway (DOT) and the mean velocity. The DOT measured the overall sway of the CoP. The mean velocity was calculated as the DOT per frame rate. The mean velocity was estimated for antero-posterior $\left(\mathrm{MV}_{\mathrm{AP}}\right)$ and medio-lateral $\left(\mathrm{MV}_{\mathrm{ML}}\right)$ directions, respectively.

\subsection{Data Analysis}

The DOT and the mean velocity (MV) parameters were estimated for each participant and for each test. The data were preliminarily screened for missing values and univariate outliers. Later, the normality assumption was verified with a Kolmogorov-Smirnov test and the visual inspection of skewness and kurtosis indexes, while the homoscedasticity was verified with Levene's test [14]. All the assumptions for univariate analysis were met. The differences between the three groups were verified by means of one-way ANOVA. If there were significant differences for a posturographic parameter, Bonferroni post-hoc test was calculated for addressing the characteristics of these differences among the groups. The effect size was estimated using the Cohen's $d$ value considering the Control group as the baseline, and it was interpreted as follows: trivial $=0-0.19$, small $=0.20-0.50$, moderate $=0.50-0.79$, and large $>0.80$ [15]. The analysis was performed separately for OE and CE test using SPSS 20.0 (SPSS Inc, Ver. 20, Chicago, IL, U.S.A.), with the significance level set to 0.05 .

\section{Results}

The analyses were conducted for each posturographic parameter for $\mathrm{OE}$ and $\mathrm{CE}$ test, separately. The differences related to DOT among the groups are shown in figure 1, while the differences related to mean velocity in antero-posterior $\left(\mathrm{MV}_{\mathrm{AP}}\right)$ and medio-lateral $\left(\mathrm{MV}_{\mathrm{ML}}\right)$ directions are shown in the figures 2 and 3 , respectively.

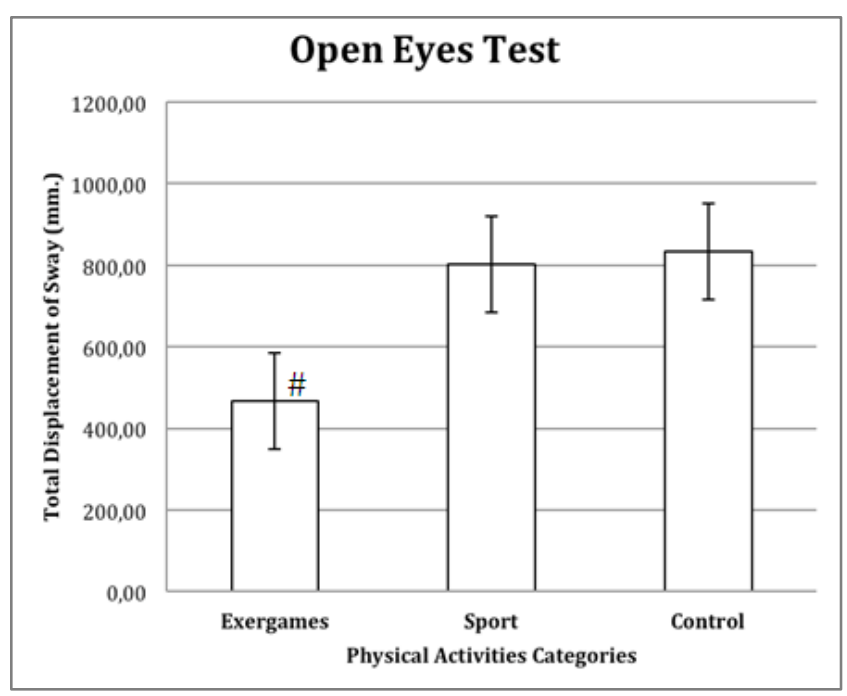

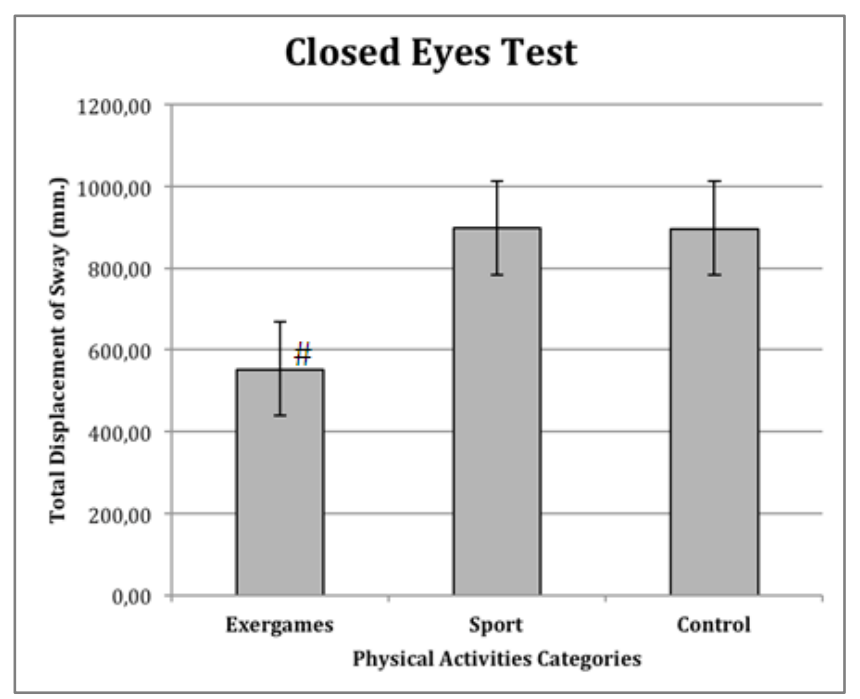

Figure 1. The differences among groups and tests for the total sway displacement parameter. The \# indicates the statistical difference $(\mathrm{p}<0.05)$ of the exergames category with the others.
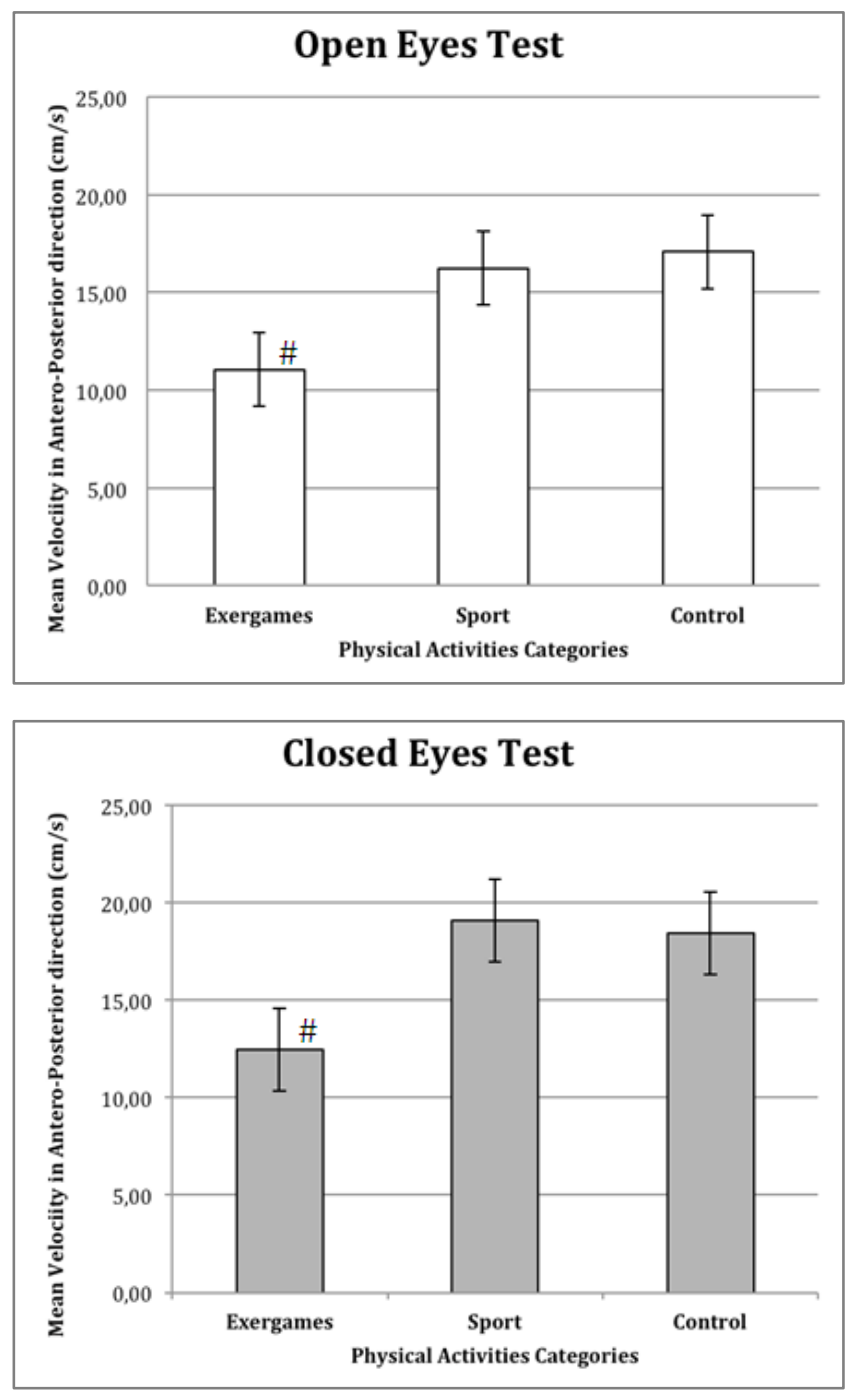

Figure 2. The differences among groups and tests for the mean velocity in antero-posterior direction. The \# indicates the statistical difference $(\mathrm{p}<0.05)$ of the exergames category with the others. 

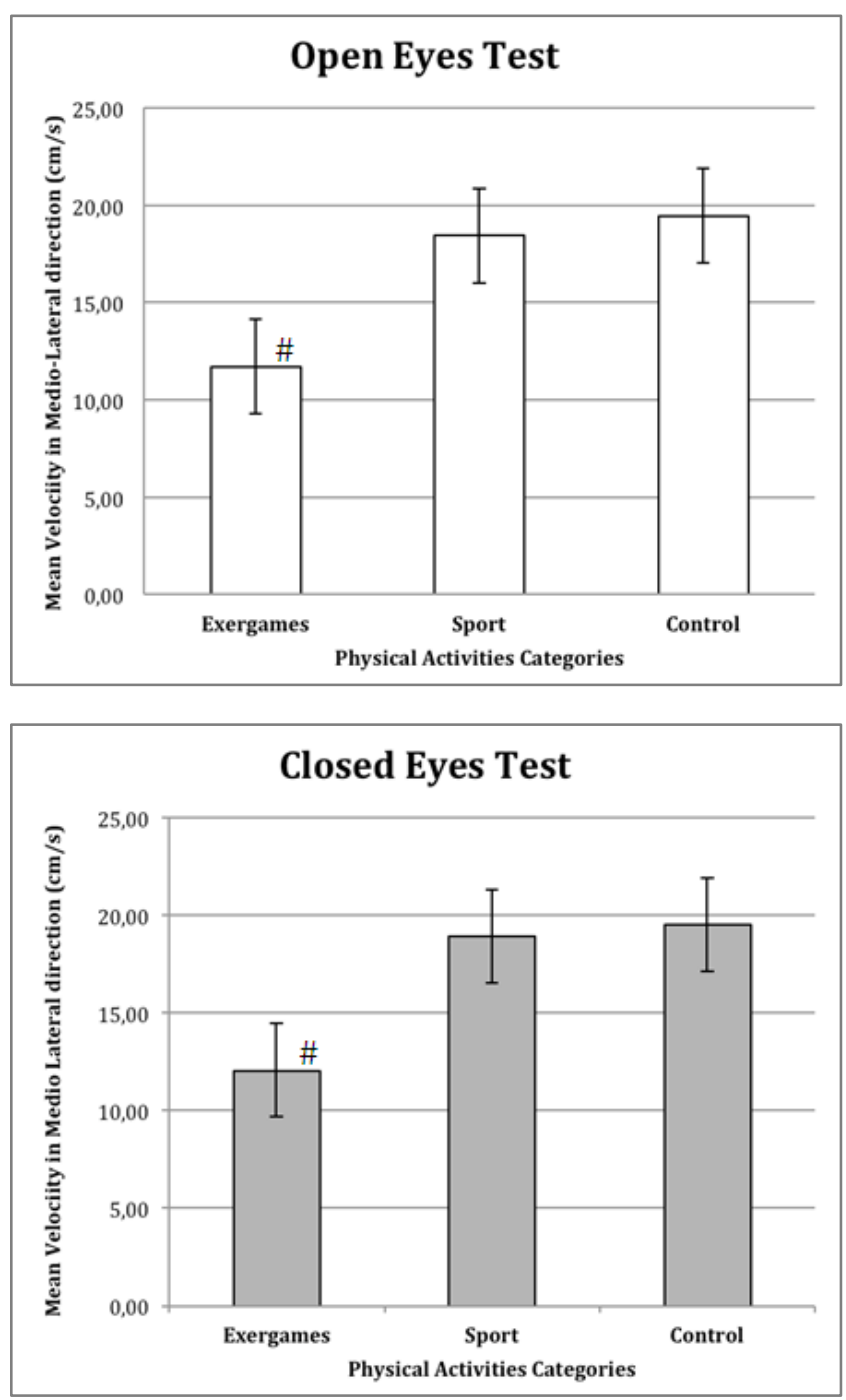

Figure 3. The differences among groups and tests for the mean velocity in medio-lateral direction. The \# indicates the statistical difference $(\mathrm{p}<0.05)$ of the exergames category with the others.

Considering the open eyes test, significant differences among the groups were found for DOT $(\mathrm{F}(2,33)=14.82$, $\mathrm{p}<.001)$, for $\mathrm{MV}_{\mathrm{AP}}(\mathrm{F}(2,33)=8.19, \mathrm{p}=.001)$, and for $\mathrm{MV}_{\mathrm{ML}}$ $(\mathrm{F}(2,33)=7.27, \mathrm{p}=.002)$. Post-hoc comparison using Bonferroni test indicated that the DOT of Exergames $(\mathrm{M}=466.10, \mathrm{SD}=283.81)$ was statistically different from Sport $(\mathrm{M}=803.02, \mathrm{SD}=74.84)$ and Control $(\mathrm{M}=833.12$, $\mathrm{SD}=121.19$ ), while no significant difference was found between Sport and Control. The effect of these differences was large between Exergames and Control (Cohen's $d=1.61$ ) and small between Sport and Control (Cohen's $d=0.30$ ). The same post-hoc test for $\mathrm{MV}_{\mathrm{AP}}$ outlined statistical difference only between Exergames $(\mathrm{M}=11.06, \mathrm{SD}=5.66)$ and Sport $(\mathrm{M}=16.24, \mathrm{SD}=2.07)$, and Control $(\mathrm{M}=17.08$, $\mathrm{SD}=3.19)$, respectively. The effect of these differences was large between Exergames and Sport (Cohen's $d=1.31$ ) and small between Sport and Control (Cohen's $d=0.31$ ). The results of post-hoc test for $\mathrm{MV}_{\mathrm{ML}}$ parameter outlined the significant differences only between Exergames $(\mathrm{M}=11.69$, $\mathrm{SD}=8.20)$ and Sport $(\mathrm{M}=18.44, \mathrm{SD}=2.33)$, and Control
$(\mathrm{M}=19.45, \mathrm{SD}=3.93)$, respectively. The estimation of the effect size suggested a large difference between Exergames and Control (Cohen's $d=1.26$ ), and a small difference between Sport and Control (Cohen's $d=0.32$ ).

Considering the closed eyes test, significant differences among the groups were found for DOT $(\mathrm{F}(2,33)=9.28$, $\mathrm{p}=.001)$, for $\mathrm{MV}_{\mathrm{AP}}(\mathrm{F}(2,33)=9.19, \mathrm{p}=.001)$, and for $\mathrm{MV}_{\mathrm{ML}}$ $(\mathrm{F}(2,33)=7.45, \mathrm{p}=.002)$. Post-hoc comparison using a Bonferroni test indicated that the DOT of Exergames $(\mathrm{M}=522.22, \mathrm{SD}=342.38)$ was statistically different from the one of Sport $(\mathrm{M}=898.00, \mathrm{SD}=114.22)$ and Control $(\mathrm{M}=886.38, \mathrm{SD}=153.64)$, while no significant difference was found between Sport and Control. The effect of these differences was large between Exergames and Control (Cohen's $d=1.21$ ) and trivial between Sport and Control (Cohen's $d=0.02$ ). The same post-hoc test for $\mathrm{MV}_{\mathrm{AP}}$ outlined statistical differences between Exergames $(\mathrm{M}=12.44, \mathrm{SD}=5.53)$ and Sport $(\mathrm{M}=19.09, \mathrm{SD}=3.33)$ and Control $(\mathrm{M}=18.44, \mathrm{SD}=3.30)$, respectively. The effect of these differences was large between Exergames and Sport (Cohen's $d=1.31$ ) and small between Sport and Control (Cohen's $d=0.20$ ). The results of post-hoc test for MV $\mathrm{ML}$ parameter outlined the significant differences between Exergames $(\mathrm{M}=12.05, \mathrm{SD}=8.05)$ and Sport $(\mathrm{M}=18.91$, $\mathrm{SD}=2.35)$ and Control $(\mathrm{M}=19.53, \mathrm{SD}=3.58)$, respectively. The estimation of the effect size provided a large difference between Exergames and Control (Cohen's $d=1.20$ ), and a small difference between Sport and Control (Cohen's $d=$ $0.20)$.

\section{Discussion and Conclusions}

The aim of this study was to investigate the influence of different physical activity training on the postural stability of university students. In this respect, we grouped the participants in three categories: Exergames, Sport, and Control. The participants were included in the Exergames if they played at least two times per week with a WBB-based exergames, while the participants were in the Sport if they were involved in structured sport activities at least two times per week. The participants in the Control were not involved in any structured physical and/or sport activities. The static postural stability was addressed by means of two standing balance tests (i.e., open and closed eyes, respectively) performed on a force-plate. The analysis of the data was based on the posturographic technique. The variables chosen for the assessment were indicated as valid and reliable in previous studies [13,21,27].

The main result of the current study was related to the differences between the Exergames and the others categories. The goodness of the exergames for supporting the training of the balance abilities was deeply recognized in previous studies [7][8]. Both studies addressed the differences between an exergames-based and a traditional balance training program, but in the first study the participants were adults, while in the second the participants were fourth grade 
school children. Gioftsidou and colleagues [16] proposed the comparison between Wii Fit-based and traditional balance training programs in Greek undergraduate students. All the previous studies confirmed the effectiveness of the exergames training programs for improving postural stability. These results were in agreement with the current ones related to the differences between the Exergames and the Control categories. In addition, the current differences between Exergames and Sport were significant. Likewise, Vernadakis and colleagues [10] suggested that an exergames-based balance training program based on the Microsoft Xbox-Kinect platform improved in significant ways the balance abilities of soccer athletes more than traditional balance training. In the current findings the Sport and the Control categories did not show significant differences for any posturographic parameters in both tests. We hypothesized that the sports played by the selected participants were not strongly oriented to the balance abilities. In this respect, previous studies showed that the balance abilities were sports dependent [4][5] and the practitioners of Basketball, American football, and Golf showed no significant differences in sway levels with non-activity control participants [24].

Considering the differences between open and closed eyes tests, the current results showed that the scores of each parameter in the OE test were better than the ones of the CE test, and they were not related to such categorization. These findings were fairly consistent with previous studies that adopted a similar methodological approach (i.e., sample characteristics, grouping, and posturographic variables) to the one used here [10][16]. The better performance of the exergames' group in absence of vision was in agreement with the interesting results obtained by Morelli and colleagues: the authors showed improvement in balance abilities of participants with visual impairments after an exergames-based training program [25]. The postural stability of the sport practitioners was better than the one of the control's students for the open-eyes test: this finding reflects the main evidence proposed in a specific review on the relation between sports and balance control [24]. In the closed eyes test, the mean velocity in the antero-posterior direction was higher in the Sport group that in the Control. This result seems to be in agreement to the findings discussed in [24] the high sway level of the soccer players compared with a non-activity group. Overall, the lack of statistical difference between Sport and Control groups for both vision tests could be explained considering that the sport practitioners (i.e. soccer athletes) use vision more for treating information that for postural stability [26]. In this respect, balance should be assessed when proprioception conditions are modified.

However, with regard to the present study, the findings were affected by some limitations. The first was related to the participants, who were selected only from a university course. A larger sample with different characteristics needs to be addressed to confirm the discussed results. Later, the Sport category was arranged without any inclusion criteria related to the different typologies of sports played by the participants. A specific analysis on the typologies of sports may be useful to better explain the differences among the categories identified in this study. Moreover, the use of a different surface (i.e. foam versus firm) will be proposed for addressing the role of proprioception in the postural stability of the selected groups.

Our analysis shows that the postural stability of the selected participants differs in terms of physical activity training. The use of exergames training programs shows interesting results for the improving of postural stability. At the same time, the exergames represents powerful tools for promoting physical activity among sedentary students. The validity and the effectiveness of this technology in physical activity, physical education, and sport topics has been debated in recent years [17][18], and we believe the students of the university course can reflect on the use of this technology in their future work activities.

\section{Acknowledgements}

We are very grateful to the participants for their support in this study.

\section{REFERENCES}

[1] Winter DA, Patla AE, Frank JS. (1990) Assessment of balance control in humans. Medical Progress through Technology. 16, 31-51.

[2] Goldie PA, Bach TM, Evans OM (1989). Force platform measures for evaluating postural control: Reliability and validity. Arch Phys Med Rehabil., 70, 510-517.

[3] Carr J.H. and Shepherd R. (1998). Neurological Rehabilitation: Optimizing Motor Performance. Oxford: Butterworth-Heinemann.

[4] Ricotti, L. (2011). Static and dynamic balance in young athletes. Journal of Human Sport and Exercise, 6(4), 616-628.

[5] Hammami, R., Behm, D. G., Chtara, M., Othman, A. B., \& Chaouachi, A. (2014). Comparison of Static Balance and the Role of Vision in Elite Athletes. Journal of Human Kinetics, 41(1), 33-41.

[6] Vernadakis, N., Gioftsidou, A., Antoniou, P., Ioannidis, D., \& Giannousi, M. (2012). The impact of Nintendo Wii to physical education students' balance compared to the traditional approaches. Computers \& Education, 59(2), 196-205.

[7] Sheehan, D. P., \& Katz, L. (2013). The effects of a daily, 6-week exergaming curriculum on balance in fourth grade children. Journal of Sport and Health Science, 2(3), 131-137.

[8] Kliem, A., \& Wiemeyer, J. (2010). Comparison of a traditional and a Video Game Based Balance Training Program. International Journal of Computer Science in Sport, 9, 80-91. 
[9] Chang, W. D., Chang, W. Y., Lee, C. L., \& Feng, C. Y. (2013). Validity and reliability of wii fit balance board for the assessment of balance of healthy young adults and the elderly. Journal of Physical Therapy Science, 25(10), 1251-1253.

[10] Vernadakis, N., Derri, V., Tsitskari, E., \& Antoniou, P. (2014). The effect of Xbox Kinect intervention on balance ability for previously injured young competitive male athletes: a preliminary study. Physical Therapy in Sport, 15(3), 148-155.

[11] Goble, D. J., Cone, B. L., \& Fling, B. W. (2014). Using the Wii Fit as a tool for balance assessment and neurorehabilitation: the first half decade of "Wii-search". $J$ Neuroeng Rehabilitation, 11(12), 3-11.

[12] Clark, R. A., Bryant, A. L., Pua, Y., McCrory, P., Bennell, K., \& Hunt, M. (2010). Validity and reliability of the Nintendo Wii Balance Board for assessment of standing balance. Gait \& Posture, 31(3), 307-310.

[13] Duarte, M., \& Freitas, S. M. (2010). Revision of posturography based on force plate for balance evaluation. Brazilian Journal of Thysical Therapy, 14(3), 183-192.

[14] Salavati M, Hadian MR, Mazaheri M, Negahban H, Ebrahimi I, Talebian S, et al. (2009) Test-retest reliability of center of pressure measures of postural stability during quiet standing in a group with musculoskeletal disorders consisting of low back pain, anterior cruciate ligament injury and functional ankle instability. Gait \& Posture, 29(3):460-464.

[15] Tabachnick, B. G. \& Fidell, L. S. (2007) Using multivariate statistics (5th ed.), Boston, MA: Allyn \& Bacon.

[16] Cohen, J. (1977) Statistical power analysis for behavioral sciences (Revised Ed.). New York: Academic Press.

[17] Gioftsidou, A., Vernadakis, N., Malliou, P., Batzios, S., Sofokleous, P., Antoniou, P., \& Godolias, G. (2013). Typical balance exercises or exergames for balance improvement. Journal of back and musculoskeletal rehabilitation, 26(3), 299-305.

[18] Robinson, J., Dixon, J., Macsween, A., van Schaik, P., \& Martin, D. (2015). The effects of exergaming on balance, gait, technology acceptance and flow experience in people with multiple sclerosis: a randomized controlled trial. BMC Sports Science, Medicine and Rehabilitation, 7(1), 8.

[19] Giblin, S., Collins, D., \& Button, C. (2014). Physical literacy: importance, assessment and future directions. Sports Medicine, 44(9), 1177-1184.

[20] Sgrò, F., Nicolosi, S., Schembri, R., Pavone, M., \& Lipoma, M. (2015). Assessing vertical jump developmental levels in childhood using a low-cost motion capture approach. Perceptual \& Motor Skills, 120(2), 642-658.

[21] Schubert, P., Kirchner, M., Schmidtbleicher, D., \& Haas, C. T. (2012). About the structure of posturography: Sampling duration, parametrization, focus of attention (part I), Journal of Biomedical Science and Engineering, 5(9), 496-507.

[22] Prieto, T. E., Myklebust, J. B., Hoffmann, R. G., Lovett, E. G., \& Myklebust, B. M. (1996). Measures of postural steadiness: differences between healthy young and elderly adults. Biomedical Engineering, IEEE Transactions on, 43(9), 956-966.

[23] Raymakers, J. A., Samson, M. M., \& Verhaar, H. J. J. (2005). The assessment of body sway and the choice of the stability parameter (s). Gait \& posture, 21(1), 48-58.

[24] Friedrich, M., Grein, H. J., Wicher, C., Schuetze, J., Mueller, A., Lauenroth, A., ... \& Schwesig, R. (2008). Influence of pathologic and simulated visual dysfunctions on the postural system. Experimental brain research, 186(2), 305-314.

[25] Schmid, M., Nardone, A., De Nunzio, A. M., Schmid, M., \& Schieppati, M. (2007). Equilibrium during static and dynamic tasks in blind subjects: no evidence of cross-modal plasticity. Brain, 130(8), 2097-2107.

[26] Kiers, H., van Dieën, J., Dekkers, H., Wittink, H., \& Vanhees, L. (2013). A systematic review of the relationship between physical activities in sports or daily life and postural sway in upright stance. Sports Medicine, 43(11), 1171-1189.

[27] Morelli, T., Liebermann, L., Foley, J., \& Folmer, E. (2014). An Exergame to Improve Balance in Children who are Blind. In Proceedings of the 9th International Conference on the Foundations of Digital Games Society for the Advancement of the Science of Digital Games. 